\title{
Sublexical units in aphasic jargon and in the standard language: Comparative analyses of neologisms in connected speech
}

\author{
Prisca Stenneken \\ Clinical Linguistics Unit, University of Bielefeld, and Freie Universität Berlin, Germany \\ Markus J. Hofmann and Arthur M. Jacobs \\ Freie Universität Berlin, Germany
}

\begin{abstract}
Background: It is a well-documented finding that phonemic speech errors in aphasia reflect certain characteristics of their intended targets. However, only few studies have investigated spontaneous speech productions of jargon-aphasic patients, in which lexical targets may be completely unrecognisable (abstruse phonemic neologisms). There is some evidence that these neologisms correspond to the standard language concerning phonemic content and structure.

Aims: The present study further explores similarities of aphasic neologisms and the standard language, to contribute to the discussion about the origin of non-target-related errors in jargon aphasia. It investigates whether similarities at the phoneme level can be confirmed even in connected, severely jargonised speech that does not allow identification of lexical targets. Moreover, it raises the question whether other sublexical units like syllables contribute to the formation of phonemic neologisms.

Methods \& Procedures: Neologisms in spontaneous speech of a German-speaking jargon-aphasic patient were compared to the standard language concerning phoneme and syllable inventory, structural aspects, and distributional frequencies of sublexical measures. Data of the standard language were derived from meta-analyses of a German phonological word form database.

Outcomes \& Results: A strong relatedness to the standard language was demonstrated for the aphasic neologisms in connected speech. Similarities regarded phoneme inventory, phonotactics, and phoneme frequency distributions. The patient data point to a preferred use of high-frequency phonemes and syllables in neologisms. In addition, a similar distribution of syllable frequencies and structures was observed in the neologisms and in standard German. Results indicate that syllable frequency serves as a predictor for the neologisms' distributional frequencies.

Conclusions: The present study indicated that phonemic neologisms with no or weak evidence for a lexical origin still conformed to the phonological characteristics of the standard language, suggesting undisturbed segmental phonological processing. With respect to processing levels in speech production, results specifically pointed to a prominent role of syllabic units. The present findings are compatible with the assumption that syllabic representations, like structural information of syllables, are constrained in neologism formation.
\end{abstract}

Keywords: Jargon aphasia; Neologism; Syllable frequency; Phoneme frequency; Syllable structure.

Address correspondence to: Prisca Stenneken, Clinical Linguistics Unit, University of Bielefeld, Postfach 1001 31, 33501 Bielefeld, Germany. E-mail: prisca.stenneken@uni-bielefeld.de

\footnotetext{
(C) 2008 Psychology Press, an imprint of the Taylor \& Francis Group, an Informa business http://www.psypress.com/aphasiology 
Speech production in jargon aphasia is typically characterised by frequent occurrence of neologistic utterances, and thus often involves a severe impairment in conveying lexical meaning. The neologistic, nonword utterances deviate from the standard language in their phonemic or semantic properties, making it difficult or impossible to identify lexical content (phonemic or semantic neologisms). With regard to phonemic neologisms, which are the focus of the present study, a distinction between two subtypes of jargon has been suggested. In neologistic jargon the nonword utterances are embedded into identifiable words or phrases, whereas phonemic jargon consists almost entirely of nonword utterances (Butterworth, 1985). Common to different forms of jargon aphasia is the observation that patients show fluent speech production, often accompanied by an auditory comprehension deficit and a failure to monitor their speech output (overview in Marshall, 2006).

Previous studies of phonemic errors in jargon have focused on the question of how far the nonword errors are related to their intended lexical targets. ${ }^{1}$ This has been a key issue in the debate concerning the origin of aphasic jargon. Findings of a high degree of target-relatedness in nonword errors (e.g., Dell, Schwartz, Martin, Saffran, \& Gagnon, 1997; Hillis, Boatman, Hart, \& Gordon, 1999) have been taken to demonstrate a lexical involvement in the neologistic productions. Thus, if similarities to the target form can be identified, the nonword error is assumed to arise from at least partial access to the target's lexical-phonological information (targetrelated error). Jargon-aphasic utterances that do not allow identification of lexical targets (so-called abstruse neologisms; Lecours, 1982) have been empirically investigated less often. This may be attributed to the relatively rarer occurrence of cases with abstruse neologism production and to the methodological challenges of having no direct comparative data set when no targets can be identified. Still, nontarget-related errors, in which the degree of lexical involvement is less obvious, present a theoretically most interesting case for investigating the processing levels that are relevant for neologism generation. In spite of the unidentifiable lexical content, jargon-aphasic speech may reflect certain characteristics of the standard language, pointing to unimpaired processing.

In general, similarities to the standard language have been reported for supralexical as well as sublexical structures in jargon. At the supralexical level, these concern normal prosodic contours and syntactic compositions (Dogil, Hildebrandt, \& Schürmeier, 1990; Duchan, Stengel, \& Oliva, 1980). At the sublexical level, jargon-aphasic errors have been repeatedly described to show a normal phoneme inventory and correspond to the phonotactic constraints of the standard language (overview in Marshall, 2006). However, the phonemic similarity between aphasic neologisms and the standard language is not undisputed (cf. Butterworth, 1979; Perecman \& Brown, 1981; Peuser \& Temp, 1981). Peuser and Temp (1981) have pointed to deviations in phoneme frequency distributions when comparing the seven most frequent phonemes of an English-speaking jargon-aphasic patient to the seven most frequent phonemes in standard English. Although these results argue

\footnotetext{
${ }^{1}$ The terminology concerning nonword errors in jargon aphasia, including neologisms and phonemic paraphasias, has not been used consistently in previous studies, especially with regard to the underlying impairment. Where no clear distinction between neologisms as non-target-related errors and phonemic paraphasias as target-related errors is made, the present study will refer to the descriptive category of nonword errors. Also, in the scope of the present study, we will use the term jargon to refer only to phonologically distorted utterances in neologistic or phonemic jargon aphasia, excluding semantic jargon.
} 
against a one-to-one correspondence in frequency ranks, the findings do not exclude a general similarity in frequency distributions. In other cases, phonemes that are not part of the standard language might also be observed when jargon aphasia is accompanied by articulatory or speech impairments. Besides, deviations in phoneme frequency distributions can occur if the aphasic jargon contains recurring utterances or repetition phenomena (a common feature of jargon), resulting in the overproportionate use of single phonemes which in turn would mask phonemic similarities to the standard language.

Concerning the empirical investigation of similarities between aphasic neologisms and the standard language, not many studies have directly addressed distributional frequencies of sublexical units. Two studies of English-speaking patients have suggested that the frequency of occurrence for phonemes reflected the phoneme frequency distribution in standard English (Hanlon \& Edmondson, 1996; Robson, Pring, Marshall, \& Chiat, 2003). With regard to syllabic processing, studies of aphasic jargon so far have focused on the sonority structures of syllables. Analyses addressed the sonority class of phonemic segments in onset and coda positions, based on the theoretical considerations of Clements (1990). Results of non-targetrelated neologisms implied that the frequency distributions of sonority structures in neologisms conformed to predictions derived from theoretic frameworks (Christman, 1992, based on the English-speaking patients MS, HV, EP) and to database corpora of the standard language (Stenneken, Bastiaanse, Huber, \& Jacobs, 2005a, based on the German-speaking patient KP). Whereas these findings were restricted to sonority structures in syllables, to our knowledge no studies are available so far that address individual syllables and their distributional frequencies.

To account for the frequent occurrence of non-target-related errors in aphasic jargon, it has been proposed that these nonword errors arise from a failure or impairment of lexical retrieval. In the case of blocked lexical retrieval, a qualitatively different, non-lexical source of nonword generation has been proposed (Buckingham, 1990; Butterworth, 1979; Kohn, Smith, \& Alexander, 1996). The resulting utterance is regarded as a substitute form for the lexical target in connected speech. Observations that the segmental structure of the nonword errors corresponded to that of real words of the standard language have been attributed to a generation mechanism that reflects the language system's inherent properties.

Under conditions of (partial) access to the target form, the nonword errors should reflect lexical content and thus also show properties of the standard language. In addition, lexical access could be followed by a severe distortion of phonemic content, involving comparable mechanisms that underlie the production of phonemic paraphasias, i.e., the addition, elision and/or substitution of one or more phonemes. It has been suggested that these distortion mechanisms could also act on an already distorted lexical retrieval and thus produce non-target-related errors (dual impairment; cf. conduction theory, Kertesz \& Benson, 1970).

Based on these considerations, the present study addressed the question of how far the non-target-related utterances in jargon aphasia reflect the characteristics of the standard language system. More specifically, the following questions were addressed: Are similarities only observed in conforming to the restrictions on phonemes and phonotactics, or is this similarity also reflected in frequency distributions? Do similarities arise at the segmental level of phonological processing, 
as suggested by previous studies? Or can other relevant sublexical units, like syllables, be identified, which reflect the properties of the standard language? To further explore possible post-lexical generation mechanisms for neologistic utterances, the present study compared phonemic and syllabic units in aphasic neologisms to those in the standard language. Analyses were based on neologistic utterances from the spontaneous speech production of the jargon-aphasic patient KP. This patient has been reported previously (Stenneken et al., 2005a), however, besides the different scope of the two studies, the present analyses were based on a different, larger data set. The present study reviews earlier results and discusses novel findings from KP. The relevant sublexical measures of the standard language were provided by a previous analysis of sublexical units in German (Hofmann, Stenneken, Conrad, \& Jacobs, 2007).

\section{METHOD}

\section{Case description}

The patient KP, a right-handed male and monolingual native German speaker, showed jargon aphasia with a frequent production of phonemic neologisms. At the age of 59 years he suffered a CVA (thrombo-embolic stroke) of the left middle cerebral artery. CT scans revealed an extensive lesion in the perfusion region of the left middle cerebral artery reaching to occipital structures. More specific case details of the patient KP have been reported previously (Stenneken et al., 2005a).

According to standardised language testing (Aachen Aphasia Test Battery; Huber, Poeck, Weniger, \& Willmes, 1983), KP's condition remained stable (testing at 5.5 and 7.5 months post-onset) and was characterised as Wernicke's aphasia with jargon. Structured language testing at 7.5 months post-onset revealed the following profile: The subtest of language comprehension was only moderately impaired, whereas severe impairments were present in all other subtests, i.e., token test, repetition, written language, and naming. There was no evidence for a speech disorder or for articulatory impairments (cf. Stenneken et al., 2005a). Accompanying deficits were ideomotor apraxia, mild right-sided facial paresis, and reduced sensibility for the right body half. Neuropsychological testing revealed deficits in visual processing and impairments in memory, learning, and attentional functions.

At the time of data collection, 7 months post-onset, spontaneous speech was fluent and utterances were interspersed with unintelligible passages. KP's speech production was characterised by the frequent occurrence of phonemic neologisms, very few phonemic paraphasias, and very rarely semantic errors. Spontaneous speech production was characterised as neologistic jargon. Here phonemic neologisms, which did not allow identifying lexical content, were embedded in short passages of intelligible speech. In contrast, KP's reading aloud was characterised as phonemic jargon, as his utterances were composed entirely of neologisms, making it impossible to identify word boundaries. In both spontaneous speech and reading aloud, KP's speech production was fluent and the intonational structure seemed adequate for German. No spontaneous self-corrections of speech errors or lexical search phenomena were observed and the patient showed no awareness that passages in his speech were incomprehensible. The identifiable utterances in KP's connected 
speech predominantly consisted of high-frequency function words, whereas nonword errors tended to be produced in slots in which content words would be expected. Examples of KP's neologistic utterances are [vot], ['ke:s-bak], [gə-'ve:-nəlt], often embedded in passages of intelligible phrases like "it was very ...", "and then we had ...", "that was my ...". Nonword errors were variable in their phonological forms, and perseverations of segments in successive syllables occurred only very rarely and no direct repetitions of neologistic syllables were observed. Nor was there any evidence for recurring utterances or utterances with a strong phonological relation to neighbouring productions. Previous analyses of KP's picture-naming performance (Stenneken et al., 2005a) supported that there was no clear evidence for a target-relatedness in KP's nonword errors. No systematic segmental correspondences between neologistic utterances and targets were observed concerning the overall number of phonemes or syllables and shared units of response and target. Thus, the neologistic jargon of KP provided a suitable data set for analyses of non-target-related errors, in which influences of lexical target forms and of neighbouring utterances were minimised.

\section{Procedure}

Speech data of KP were collected on two consecutive days in two sessions of about 1 hour each. Neologisms were elicited in guided spontaneous speech samples in a semi-structured interview on familiar themes and in descriptions of coloured drawings of everyday situations. The conversation was tape recorded and transcribed orthographically. All utterances that deviated from standard German (nonword errors) were phonetically transcribed by one trained coder and checked for accuracy by two others. The nonword utterances, enclosed in utterances of intelligible speech, had a length of one to three syllables. Sublexical frequency measures for KP's neologistic utterances were determined for single and dual phonemic units (phonemes and biphonemes), and for syllabic units, by summing up the frequency of occurrence for each of the units in the sample of neologistic utterances. Type frequency measures were calculated by counting the amount of words containing the respective phonemic or syllabic unit. For example, the type frequency of the syllable "ba" denotes the number of utterances that contain this unit as a syllable. In order to compare the characteristics of the aphasic errors to the standard language, the German CELEX phonological word form database (Baayen, Piepenbrock, \& Gulikers, 1995) was selected as a reference base. Sublexical frequency measures of the standard language were computed for phonemes, biphonemes, and syllables (according to Hofmann et al., 2007). In addition, token frequencies were computed by summing up the lexical frequencies of the words that contain the respective sublexical unit. For example, token frequencies for the syllable "ba" were determined by adding up the word frequencies for all words possessing the syllable "ba". Lexical frequency measures were taken from the CELEX's Mannheim frequency counts in words per 6 million. All following analyses of the standard language are based on the token frequencies of the sublexical measures. More detailed information about corpus analyses and applied algorithms can be examined elsewhere (Hofmann et al., 2007). Preliminary analyses of a smaller subset of data from KP's spontaneous speech production data have been previously reported (Stenneken, Hofmann, \& Jacobs, 2005c). 


\section{RESULTS}

\section{Phoneme analyses}

The data set of KP's neologistic utterances comprised 1643 phonemic units, which corresponded to 38 different German phonemes (when distinguishing tense and lax vowels). All segments were part of the German phoneme inventory and their combinations conformed to German phonotactics. Phoneme frequency analyses compared the frequencies of occurrence for the phonemes in the aphasic data to phoneme frequencies in the normative data. The two data sets were highly correlated $(r=.91, p<.001)$. Thus, phonemes with a high frequency in German tended to occur more frequently in the aphasic data and those with a low frequency in German tended to occur less frequently (Figure 1). In particular, the distributions suggested a numerical tendency to produce high-frequency phonemes in KP. When assigning German phoneme frequencies to logarithmic frequency classes (class 1 starting from 10,000 occurrences, 2 starting from 100,000, 3 starting from 1,000,000), only $3 \%$ of the phonemes produced by KP fell into the lowest frequency class. About $47 \%$ of the phonemes were in the medium frequency range, and $48 \%$ fell into the highest frequency class. In contrast, relatively fewer phonemes in the standard data belonged to the highest frequency class (18\% in frequency class $1,66 \%$ in class 2 , and $15 \%$ in class 3).

Additionally, to address dual units of phonemes, correlation analyses were performed on biphoneme frequencies, i.e., the frequencies of occurrence for biphonemes in the neologistic data and biphoneme frequencies in the normative data. Again, results showed that the two data sets were highly correlated $(r=.36$, $p<.001$ ), pointing to preferred occurrences of high-frequency dual units of phonemes in the aphasic neologisms, when the biphonemes were of high frequency in the standard language.

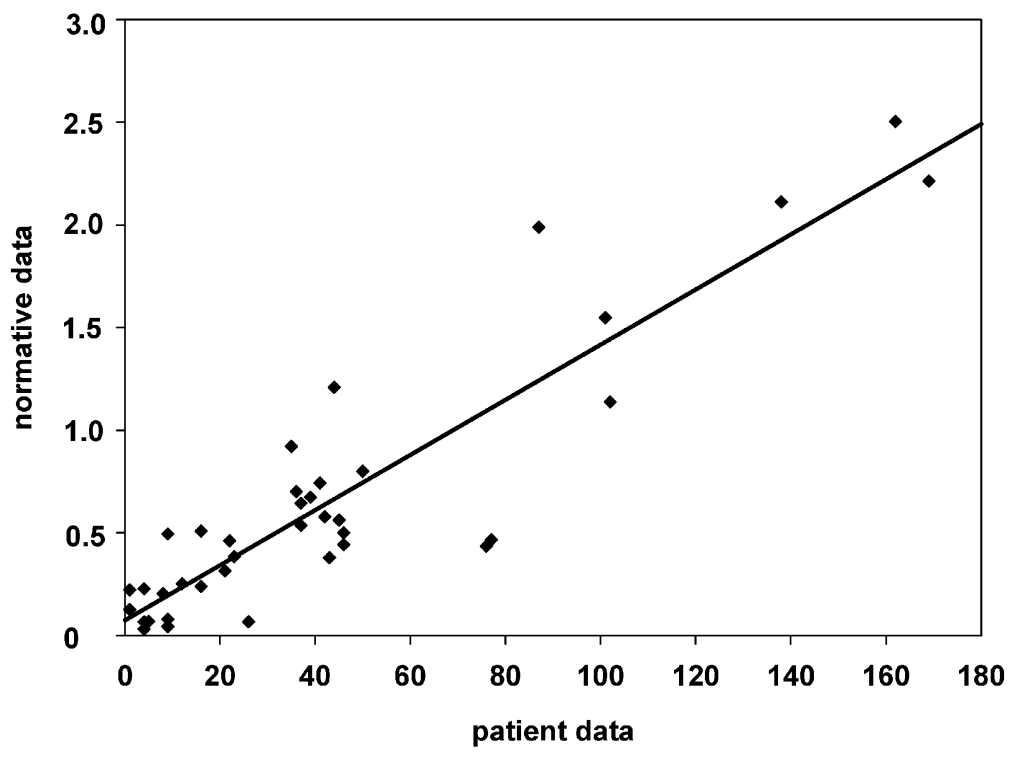

Figure 1. Scatterplot of phoneme frequencies in nonword errors of the aphasic jargon (patient data) and those in German (normative data: occurrences in millions). 
Discussion. The present results showed that jargon-aphasic neologisms consisted of phonemes of the standard language and complied with its phonotactics. These findings from connected speech are in line with those of previous studies using a variety of language tasks (Buckingham \& Kertesz, 1976; Butterworth, 1979; Christman, 1994; Duchan et al., 1980; Robson et al., 2003). Moreover, KP's neologisms showed a distribution of phoneme frequencies similar to that in the standard language. A comparable, though weaker, correlation was observed for biphonemes. In general, the findings point to a strong role of phonemic units in neologism formation. Comparable results for phoneme frequencies have been reported in one study of spontaneous speech utterances in an English-speaking jargon-aphasic patient (Hanlon \& Edmondson, 1996). Other studies have shown an influence of phoneme frequency in jargon-aphasic naming errors (Robson et al., 2003) and in errors produced by aphasic patients without jargon in both spontaneous and structured speech tasks (Gordon, 2002). Together with the tendency in KP to produce high-frequency phonemes, the present findings suggest a strong influence from segmental phonology of the standard language on abstruse neologism generation. More specifically, phoneme inventory, phoneme combinations, and frequencies in KP point to undisturbed segmental phonology, even in jargon that does not show a clear influence of lexical targets or of neighbouring utterance units.

\section{Syllable analyses}

So far, analyses of aphasic neologisms focused on phonemic units, whereas little is known about the relevance of other sublexical units like syllables. The present analyses therefore compared the syllables in KP's neologisms to those of the standard language concerning the measures of syllable inventory, frequency distributions, and syllable structure. The data set of KP's neologisms comprised 600 syllabic units that corresponded to 310 different syllable types.

A comparison of the syllables in the aphasic data to the syllable inventory of the German database revealed that 47 of KP's syllables (corresponding to about $15 \%$ of the data) did not occur in the standard language, although all were phonologically legal. Further analyses determined the frequencies of occurrence for the syllables in KP's nonword errors and compared these to syllable token frequencies in the standard data. Results showed a significant positive correlation $(r=.600, p<.001)$, indicating similar distributions of syllable frequencies in the two data sets (Figure 2). In addition, the distributional pattern pointed to a preferential use of high-frequency syllables in KP's neologisms. Logarithmic token frequency classes of syllables in the standard language were formed (i.e., occurrences of 10, 100, 1000, 10,000, and so on in German phonological word forms). In the neologism data, more syllables fell into higher logarithmic frequency classes. Results showed a continuous rise, with fewest syllables of KP (3\%) belonging to the lowest frequency class, followed by $6 \%, 14 \%$, $25 \%, 34 \%$ with increasing frequency classes. This observation did not extend to the highest logarithmic frequency class with only $9 \%$ of KP's syllables. However, this class of German syllables comprised only 11 different units, mostly representing monosyllabic function words. In contrast to the patient data, most German syllables belong to the lower frequency classes ( $35 \%$ to the lowest class, followed by $26 \%, 23 \%$, and only $16 \%$ for all remaining high-frequency classes). 


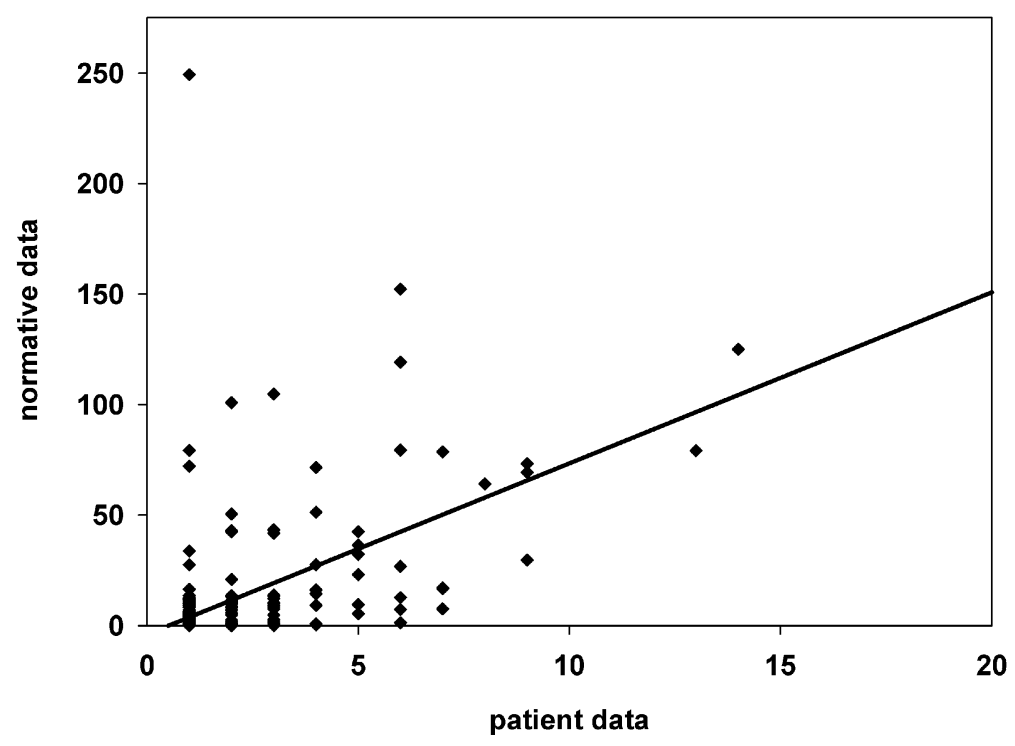

Figure 2. Scatterplot of syllable frequencies in nonword errors of the aphasic jargon (patient data) and those in German (normative data: occurrences in thousands).

As second syllabic measure, syllable complexity was determined for a data set of 277 syllables in KP's neologisms, which had been classified according to their sonority structures in previous analyses (Stenneken et al., 2005a). For the present purpose, the following complexity categories were derived for syllable onset and coda positions: no consonantal segments in onset or coda position (single vowel, V), single consonant in onset or coda (CV or VC), or clusters of two or three consonants (CCV or VCC, CCCV or VCCC). Clusters of more than three consonants made up only $0.5 \%$ of KP's data and therefore did not enter analyses. Results indicate that the distributions of syllable complexity types were also highly similar in the aphasic neologisms and in the standard language (Figure 3).

Discussion. The analyses of syllabic units have again revealed similarities between the aphasic neologisms and the standard language. These concerned the frequency distributions of syllables and syllable structures. Supported by a preferred use of high-frequency syllables in the aphasic neologisms, the present findings suggest a relevance of syllabic units in neologism formation.

One observation that seemed to contradict a linear relation of the two data sets may be discussed in more detail. The syllable /di/ has a rather low frequency in KP but an extraordinarily high frequency in standard German (about 250,000 occurrences; see Figure 2). This phonological unit not only occurs as a syllable in many German words but also corresponds to the feminine definite article (die) and thus also has a high word frequency. The observed results could therefore be due to the methodological procedure, in which identifiable real-word utterances in the patient data did not enter the analyses, leading to the exclusion of syllables that could also represent monosyllabic words. Thus this observation suggests - as has also been observed for phoneme frequencies - that the similarity in the distributions of the patient and the standard data express a general tendency but not a one-to-one equivalence in frequency ranks. 


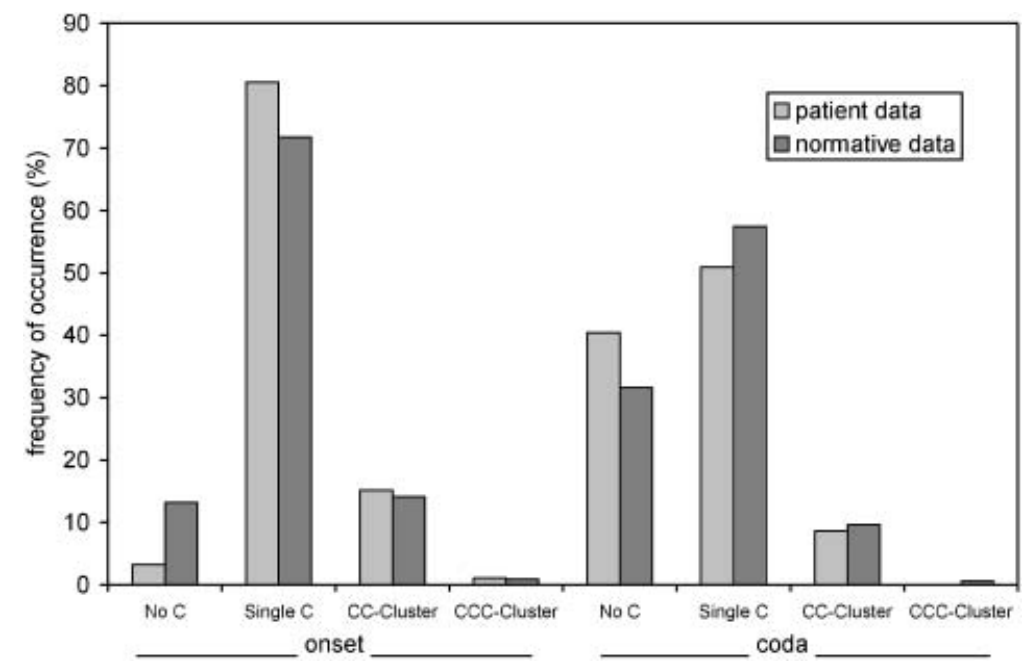

Figure 3. Distribution of syllable structures in nonword errors of the aphasic jargon (patient data) and in German (normative data) according to types of onset complexity (left side) and coda complexity (right side). $(\mathrm{C}=$ consonant in onset or coda positions, $\mathrm{CC}=$ double consonants, $\mathrm{CCC}=$ cluster of three consonants.)

Concerning syllable frequencies in aphasic neologisms, not much is known from previous studies. There is some evidence for syllable frequency effects from other language impairments, including aphasic patients with phonological production or reading deficits (Laganaro, 2005; Staiger, Ziegler, \& Schmid, 2006; Stenneken, Conrad, Hutzler, Braun, \& Jacobs, 2005b) or patients with apraxia of speech (Aichert \& Ziegler, 2004a; Laganaro, 2008 this issue; Staiger \& Ziegler, 2008 this issue). Here, the syllable frequency of target stimuli was shown to affect error rates or reaction times in a variety of tasks including repetition, reading aloud, visual lexical decision, and naming in German and French (but see Wilshire \& Nespoulous, 2003, for null effects in two French-speaking aphasic patients). One of these studies (Staiger et al., 2006) focused on spontaneous speech productions and demonstrated that patients with aphasia showed a preferred use of high-frequency syllables in their spontaneous speech utterances.

The observations concerning syllable frequencies can be related to findings on syllable structures. The present results showed similar distributions of consonants in the syllable structures of aphasic neologisms and standard data. Consistent findings have been reported for sonority structures (Christman, 1992, 1994). Results indicated that syllabic sonority structures in non-target-related errors of jargonaphasic patients conformed to the predictions for the standard language as derived from theory (Christman, 1992, based on Clements, 1990). Furthermore, when comparing target-related errors to their intended targets, the aphasic utterances showed either unchanged or decreased syllable complexity concerning the sonority profiles (Christman, 1994; for a similar tendency to theoretically preferred sonority profiles in connected speech, see previous analyses of KP in Stenneken et al., 2005a). Supportive evidence for a role of syllable structures, constrained by preferred sonority contours, in speech production comes from aphasia syndromes without jargon (Bastiaanse, Gilbers, \& van der Linde, 1994; Den Ouden, 2002; Romani \& Calabrese, 1998). 
Based on the empirical findings of syllable frequency effects, it has been discussed whether phonological processing involves the retrieval of syllable-sized representations (Cholin, 2008 this issue; Conrad, Stenneken, \& Jacobs, 2006; Laganaro \& Alario, 2006). Although the results of the present study point to syllables as relevant processing units, the interpretation may be limited by the finding that, in the speech sample of patient KP, $15 \%$ of the syllabic units, all legal phoneme combinations like [tsom] or [lغçt], did not occur as syllables in the standard language database. This observation argues against the assumption that KP's nonword errors are generated from chains of syllabic units, as has been suggested in a related way for phonemic units in abstruse neologism formation (cf. Buckingham, 1990). Rather, the finding of new-formed syllables would be compatible with the assumption that syllabic representations influenced other sublexical (e.g., segmental) processing domains. Supportive empirical evidence for an influence of other phonological processing domains on segmental processing comes from a patient with phonological jargon (Janßen \& Domahs, 2008 this issue). Here, error analyses indicated that segmental processing was influenced by metrical processing. In the following section the different sublexical processing domains and their possible contributions will be further explored by additional analyses.

\section{Regression analyses}

A multiple linear regression analysis was conducted that took different sublexical measures into account. It explored whether a sublexical unit in the standard language can be identified that best predicts the frequency of occurrence for the aphasic nonword errors. The frequency of occurrence of KP's neologistic syllables served as criterion variable, and sublexical predictor variables were phoneme frequency, biphoneme frequency, and syllable frequency in the standard language. For each utterance unit in the patient data, the corresponding sublexical measures in the standard language were computed from the German word form database (Baayen et al., 1995) as syllable frequencies and as averaged frequencies for all phonemes or biphonemes occurring in the aphasic utterances. Since no word boundaries could be determined in the neologistic jargon, utterances were separated into syllabic units for the present analyses. The data set comprised 305 syllabic units in KP's neologisms, having excluded five units that consisted of a single vowel so that biphoneme frequencies could not be computed.

A significant model emerged, $F(3,301)=58.56, p<.001$; adjusted $R^{2}=.362$. Results were significant only for syllable frequency $(t=10.70, p<.001)$, whereas biphoneme and phoneme frequency were not a significant predictor in this model (biphoneme frequency: $t=1.40, p=.164$; phoneme frequency: $t=0.29, p=.772$ ). With regard to the method of computing the different sublexical measures, these cannot be assumed to be completely independent of other. Additional analyses revealed a low correlation of syllable frequency and phoneme frequency $(r=.18$, $p<.001)$, whereas syllable frequency and biphoneme frequency were moderately correlated $(r=.47, p<.001)$.

Discussion. The results from regression analysis suggest that the averaged frequencies of phonemes and biphonemes, and the frequencies of syllables in the standard language, did not each contribute independently to the frequency of occurrence of KP's neologistic utterances; rather, only syllable frequency served as 
predictor for the frequency pattern of the aphasic utterances. Syllable frequency accounted for around $36 \%$ of the variance in the frequency of occurrence of the neologistic utterances. This finding, that syllable frequency is the factor that is crucially responsible for the variance in the aphasic data, supports a role of syllabic units in neologism formation. The present analyses did not include syllable structures, since these did not contain segmental information. Still, as stated above, the observed effect of syllable frequency would also be compatible with a relevance of syllables' structural information, since syllables with a higher frequency of occurrence also tend to have less complex syllable structures.

Moreover, the assumption of a prominent role of syllabic units does not contradict the observed similarities in phoneme frequency distributions for the aphasic and the normative data. Again, a correlation between the measures of phoneme and syllable frequencies would be expected, since a high frequency of occurrence for larger units, like words or syllables, is also reflected in a high frequency of constituent units, like phonemes. In general, it is hard to disentangle phoneme (or biphoneme) and syllable frequency effects. When a given utterance is segmented into phonemes, more observations are available than when the same utterance is segmented into syllables. In multiple regression analyses using phoneme and syllable frequency predictors, phoneme frequencies were averaged, whereas syllable frequencies were entered without prior transformation. ${ }^{2}$ In general, the present methodological considerations point to the relevance of addressing different sublexical measures in the analyses of aphasic data (see Aichert \& Ziegler, 2005; Hofmann, et al., 2007). To summarise, the present data seem to be best explained by assuming a prominent role of syllabic units in neologism formation. In the following section this interpretation will be discussed in more detail and related to the underlying deficit in KP.

\section{GENERAL DISCUSSION}

The present study revealed similarities in aphasic neologisms and the standard language for different sublexical measures. The results of the comparative analyses can be interpreted as follows: (1) Undisturbed segmental phonology can be assumed even for neologisms in which lexical influence is minimised. (2) Sublexical information, based on representations of syllabic units or structures of the standard language, seems to influence neologism formation.

With regard to the first assumption, evidence was provided by the phoneme inventory and the compliance with German phonotactics in the patient's neologisms. In addition, phonological processing in KP seemed to reflect the standard language's phoneme frequencies, in that phonemes of high frequency in German had a higher chance of being produced in the neologisms than those of lower frequencies. The present study extended previous evidence from English-speaking patients (e.g., Hanlon \& Edmondson, 1996) for intact phonological processes in jargon aphasia by findings from an additional phonology, German, that allows for rather complex

\footnotetext{
${ }^{2}$ Comparable findings were obtained in regression analyses that alternatively employed the minima of phoneme, biphoneme, and syllable frequencies, $F(3,301)=59.66, p<.001$; adjusted $R^{2}=.367$. Results still indicated that syllable frequency was the best predictor $(t=7.23, p<.001)$. In addition, the results were significant for biphoneme frequency $(t=2.09, p=.038)$; however, this measure might not have a strong individual contribution as it appeared to be highly correlated with syllable frequency $(r=.74, p<.001)$.
} 
phonological structures. In sum, results from phoneme analyses supported the notion that phonemes are functionally relevant production units in neologism formation. In addition to most previous phonological investigations of aphasic nonword errors, the present study also demonstrated phonemic similarities for utterances in which lexical influence can be assumed to be minimised. The assumption of a lexical impairment in KP was confirmed by several aspects in his language production. In connected speech, hardly any phonemic paraphasias were observed that would have pointed to a phonemic distortion of lexical targets. Neologisms were embedded in passages of intelligible speech and tend to occur in slots in which a content word would be expected, compatible with the notion of neologisms as surrogate forms in case of impaired lexical access. Similarly, in structured language tasks, there was no clear evidence for a phonemic relatedness of KP's responses to their targets (Stenneken et al., 2005a). In general, neologisms were variable in their phonological form and inconsistent responses are made to identical items, arguing against a deficient lexical representation. In sum, this pattern in the speech production of KP would be compatible with the association of neologism formation to impaired lexical retrieval or lost phonological information (cf. Buckingham \& Kertesz, 1976; Butterworth, 1979) and the implication that segmental phonology would proceed disconnected from lexical processing (Hanlon \& Edmondson, 1996).

An alternative interpretation of KP's disorder would be to assume a lexical origin of the neologism formation, in which word forms are phonemically distorted after lexical selection. Here, lexical selection can either be assumed to be unimpaired or to involve errors in selecting the wrong form (discussion in Robson et al., 2003). Since phoneme frequency is marked not only at the phoneme but also on the lexical level, the observed similarities in the neologism to the standard language might also reflect the phonemic structure of a lexical target. On the basis of the present data, this interpretation cannot be excluded as the source of KP's neologism generation. However, with regard to completely incomprehensible neologistic utterances, a severe distortion of the phonological form of the lexical item must be assumed, so that the target's phonemic structure is not evident from the neologistic utterance. It is not possible to finally decide between these two alternative accounts of neologism formation-both would allow for the conclusion that the observed similarity of the aphasic errors to the standard language could hardly be attributed to the phonemic content of a certain lexical target.

The second, novel aspect of the present investigations regarded the role of syllabic units in neologism formation. So far, evidence for undisturbed sublexical processing mainly concerned segmental phonological processing. The present findings indicated that syllable frequencies are also distributed similarly in neologisms and in standard German. Moreover, a strong relevance of syllables in neologism generation was suggested by the effects of syllable frequency, i.e., finding that syllable frequency in the standard data served as the predictor of the frequencies of the jargon-aphasic utterances.

On the one hand, the present findings would be compatible with the assumption that syllables are individually represented and retrieved in speech production. A specific view has been proposed by the concept of the syllabary, from which gestural scores for high-frequency syllables are assumed to be accessed during phonetic encoding (Levelt, Roelofs, \& Meyer, 1999; Levelt \& Wheeldon, 1994). On the other hand, the interpretation is restricted by an observation concerning syllable types. As 
discussed above, the finding of $15 \%$ new-formed syllables in the aphasic neologisms makes it unlikely that neologisms are composed as chains of syllabic units from the standard language. Rather, the findings from syllable structure and syllable complexity allow for an alternative view, according to which the observed effect of syllable frequency might result from structural syllable computation. Assuming a preference for non-complex syllables, the emerging utterances should also show a tendency for higher-frequent syllables. In more general terms, syllabic representations might play a role in neologism formation in that it constrains processing in other sublexical domains. The observation that the phonemic sequences in KP deviated from those of lexical forms can be related to the notion of an indexing failure while accessing the syllable nodes, which has been suggested to underlie speech errors in normal language production (Levelt et al., 1999; for a related account of segmental errors in an aphasic speaker, see Aichert \& Ziegler, 2004b).

Moreover, the above considerations are compatible with models of speech production that assume an explicit representation of the structural information of syllables (cf. Dell, 1988; Shattuck-Hufnagel, 1992). This framework, in contrast to models that assume access to represented syllabic units or gestures, has been directly related to neologism production in aphasia (Dell et al., 1997; Hillis et al., 1999, Robson et al., 2003). It has been proposed that neologisms arise from a global weakening of connections in the model's connectionist architecture (Schwartz, Saffran, Bloch, \& Dell, 1994) or from a local impairment of the connections between the lexical and the phonological level (Hillis et al., 1999). Both views would predict a reduced influence from the lexical level on the phonological nodes, which would facilitate the selection of non-target segmental information. Moreover, this type of model can account well for an additional observation in the present study, i.e., the tendency of KP's neologisms to include high-frequency sublexical units. Assuming that phonemes or syllable structures with high frequency in the standard language need less activation to reach the selection threshold as compared to units of lower frequencies, the model would predict a stronger tendency to produce high-frequency units the weaker the influence from the lexical nodes.

In general, the present study suggested that neologism generation is determined by sublexical characteristics of the standard language, even for connected jargonised speech that does not allow identification of lexical targets. More specifically, results point to a prominent role of syllabic representations that are unaffected by a lexical impairment and may influence segmental processing in neologism generation. Finally, findings from both phoneme and syllable analyses are compatible with an increased relevance of the standard language's sublexical principles when lexical influences are minimised.

\section{REFERENCES}

Aichert, I., \& Ziegler, W. (2004a). Syllable frequency and syllable structure in apraxia of speech. Brain and Language, 88, 148-159.

Aichert, I., \& Ziegler, W. (2004b). Segmental and metrical encoding in aphasia: Two case reports. Aphasiology, 18, 1201-1211.

Aichert, I., \& Ziegler, W. (2005). Is there a need to control for sublexical frequencies? Brain and Language, $95,170-171$.

Baayen, R. H., Piepenbrock, R., \& Gulikers, L. (1995). The CELEX lexical database [CD-ROM]. Philadelphia: Linguistic Data Consortium, University of Pennsylvania. 
Bastiaanse, R., Gilbers, D., \& van der Linde, K. (1994). Sonority substitutions in Broca's and conduction aphasia. Journal of Neurolinguistics, 8, 247-255.

Buckingham, H. W. (1990). Abstruse neologisms, retrieval deficits and the random generator. Journal of Neurolinguistics, 5, 215-235.

Buckingham, H. W., \& Kertesz, A. (1976). Neologistic jargon aphasia. Amsterdam: Swets \& Zeitlinger.

Butterworth, B. (1979). Hesitation and the production of verbal paraphasias and neologisms in jargon aphasia. Brain and Language, 8, 133-161.

Butterworth, B. (1985). Jargon aphasia: Processes and strategies. In S. Newman \& R. Epstein (Eds.), Current perspectives in dysphasia (pp. 321-352). Edinburgh, UK: Churchill Livingstone.

Cholin, J. (2008). The mental syllabary in speech production: An integration of different approaches and domains. Aphasiology, 22, 1127-1141.

Christman, S. S. (1992). Uncovering phonological regularity in neologisms: Contributions of sonority theory. Clinical Linguistics and Phonetics, 6, 219-247.

Christman, S. S. (1994). Target-related neologism formation in jargonaphasia. Brain and Language, 46, $109-128$.

Clements, G. N. (1990). The role of sonority in core syllabification. In J. Kingston \& M. E. Beckman (Eds.), Papers in laboratory phonology I. Between the grammar and physics of speech (pp. 283-333). Cambridge, UK: Cambridge University Press.

Conrad, M., Stenneken, P., \& Jacobs, A. M. (2006). Associated or dissociated effects of syllable frequency in lexical decision and naming. Psychonomic Bulletin \& Review, 13, 339-345.

Dell, G. S. (1988). The retrieval of phonological forms in production: Tests of predictions from a connectionist model. Journal of Memory and Language, 27, 124-142.

Dell, G. S., Schwartz, M. F., Martin, N., Saffran, E. M., \& Gagnon, D. A. (1997). Lexical access in aphasic and nonaphasic speakers. Psychological Review, 104, 801-838.

den Ouden, D. B. (2002). Segmental vs syllable markedness: Deletion errors in the paraphasias of fluent and non-fluent aphasics. In E. Fava (Ed.), Clinical linguistics: Theory and applications in speech pathology and therapy. Current issues in linguistic theory 227 (pp. 23-45). Amsterdam: John Benjamins.

Dogil, G., Hildebrandt, G., \& Schürmeier, K. (1990). The communicative function of prosody in a semantic jargon aphasia. Journal of Neurolinguistics, 5, 353-369.

Duchan, J. F., Stengel, M. L., \& Oliva, J. (1980). A dynamic model derived from the intonational analysis of a jargon aphasic patient. Brain and Language, 9, 289-297.

Gordon, J. K. (2002). Phonological neighborhood effects in aphasic speech errors: Spontaneous and structured contexts. Brain and Language, 82, 113-145.

Hanlon, R., \& Edmondson, J. A. (1996). Disconnected phonology: A linguistic analysis of phonemic jargon aphasia. Brain and Language, 55, 199-212.

Hillis, A., Boatman, D., Hart, J., \& Gordon, B. (1999). Making sense out of jargon: A neurolinguistic and computational account of jargon aphasia. Neurology, 53, 1813-1824.

Hofmann, M. J., Stenneken, P., Conrad, M., \& Jacobs, A. M. (2007). Sublexical frequency measures for orthographic and phonological units in German. Behavior Research Methods, 39(3), 620-629.

Huber, W., Poeck, K., Weniger, D., \& Willmes, K. (1983). Aachener Aphasie Test. Göttingen: Hogrefe.

Janßen, U., \& Domahs, F. (2008). Going on with optimised feet: Evidence for the interaction between segmental and metrical structure in phonological encoding from a case of primary progressive aphasia. Aphasiology, 22, 1157-1175.

Kertesz, A., \& Benson, D. (1970). Neologistic jargon: A clinicopathological study. Cortex, 6, 362-386.

Kohn, S., Smith, K., \& Alexander, M. (1996). Differential recovery from impairment to the phonological lexicon. Brain and Language, 52, 129-149.

Laganaro, M. (2005). Syllable frequency effect in speech production: Evidence from aphasia. Journal of Neurolinguistics, 18, 221-235.

Laganaro, M. (2008). Is there a syllable frequency effect in aphasia or in apraxia of speech or both? Aphasiology, 22, 1191-1200.

Laganaro, M., \& Alario, F.-X. (2006). On the locus of the syllable frequency effect in speech production. Journal of Memory and Language, 55, 178-196.

Lecours, A. R. (1982). On neologisms. In J. Mehler, S. Franck, E. Walker, \& M. Garret (Eds.), Perspectives of mental representation (pp. 217-247). Hillsdale, NJ: Lawrence Erlbaum Associates Inc.

Levelt, W. J. M., Roelofs, A., \& Meyer, A. S. (1999). A theory of lexical access in speech production. Behavioral and Brain Sciences, 22, 1-75.

Levelt, W. J. M., \& Wheeldon, L. (1994). Do speakers have access to a mental syllabary? Cognition, 50, 239-269. 
Marshall, J. (2006). Jargon aphasia. What have we learned? Aphasiology, 20, 387-410.

Perecman, E., \& Brown, J. W. (1981). Phonemic jargon: A case report. In J. W. Brown (Ed.), Jargonaphasia (pp. 177-258). New York: Academic Press.

Peuser, G., \& Temp, K. (1981). The evolution of jargon aphasia. In J. W. Brown (Ed.), Jargonaphasia (pp. 259-294). New York: Academic Press.

Robson, J., Pring, T., Marshall, J., \& Chiat, S. (2003). Phoneme frequency effects in jargon aphasia: A phonological investigation of nonword errors. Brain and Language, 85, 109-124.

Romani, C., \& Calabrese, A. (1998). Syllabic constraints in the phonological errors of an aphasic patient. Brain and Language, 64, 83-121.

Schwartz, M., Saffran, E., Bloch, D., \& Dell, G. (1994). Disordered speech production in aphasic and normal speakers. Brain and Language, 47, 52-88.

Shattuck-Hufnagel, S. (1992). The role of word structure in segmental serial ordering. Cognition, 42, 213-259.

Staiger, A., \& Ziegler, W. (2008). Syllable frequency and syllable structure in the spontaneous speech production of patients with apraxia of speech. Aphasiology, 22, 1201-1215.

Staiger, A., Ziegler, W., \& Schmid, G. (2006). Frequency and structure of sublexical units in the spontaneous speech production of apraxic and aphasic patients. Presentation at the Science of Aphasia Meeting VII, Porto Conte, Italy.

Stenneken, P., Bastiaanse, R., Huber, W., \& Jacobs, A. M. (2005a). Syllable structure and sonority in language inventory and aphasic neologisms. Brain and Language, 95, 280-292.

Stenneken, P., Conrad, M., Hutzler, F., Braun, M., \& Jacobs, A. M. (2005b). Frequency effects with visual words and syllables in a dyslexic reader. Behavioural Neurology, 16, 103-117.

Stenneken, P., Hofmann, M., \& Jacobs, A. M. (2005c). Patterns of phoneme and syllable frequency in jargon aphasia. Brain and Language, 95, 221-222.

Wilshire, C. E., \& Nespoulous, J. L. (2003). Syllables as units in speech production: Data from aphasia. Brain and Language, 84, $424-447$. 
Copyright of Aphasiology is the property of Psychology Press (UK) and its content may not be copied or emailed to multiple sites or posted to a listserv without the copyright holder's express written permission. However, users may print, download, or email articles for individual use. 